\title{
Electric Conduction in a Single GaN Nanocolumn*
}

\author{
Yuya Kato, Youhei Nakata, Haruhiko Kuroe, and Tomoyuki Sekine ${ }^{\dagger}$ \\ Department of Physics, Sophia University, Tokyo 102-8554, Japan \\ Akihiko Kikuchi and Katsumi Kishino \\ Department of Electrical and Electronics Engineering, Sophia University, Tokyo 102-8554, Japan \\ Nobuyuki Aoki and Yuichi Ochiai \\ Graduate School of Advanced Integration Science, Chiba University, Chiba 263-8522, Japan
}

(Received 1 February 2012; Accepted 31 May 2012; Published 14 July 2012)

\begin{abstract}
We study electric conduction of single GaN nanocolumns, which are synthesized by rf-plasma-assisted molecular beam epitaxy. We attach $\mathrm{Ti} / \mathrm{Al}$ electrodes to a single $\mathrm{GaN}$ nanocolumn on $\mathrm{SiO}_{2} / \mathrm{Si}$ substrate by using photolithography, and we succeed in creating the ohmic contacts. We observe the nonlinear conduction at large currents and low temperatures. The temperature dependence of resistivity represents the activation-type one with an activation energy of about $11 \mathrm{meV}$ due to donor levels at high temperatures. At low temperatures, it shows the Mott variable-range hopping conduction. We find a strong positive magnetoresistance effect at low temperatures. [DOI: $10.1380 /$ ejssnt.2012.355]
\end{abstract}

Keywords: Electric conductivity; Magnetoresistance; Gallium nitride; Nanocolumns

\section{INTRODUCTION}

Because the semiconductor columnar nanostructures, i.e., the semiconductor nanocolumns have an excellent light-emitting property, it is expected to become one of the next generation of the optical nanodevices. It provides big possibilities of not only applications to nanodevices but also emergence of novel physical phenomena. In particular, GaN and the related nitrides are highly attractive materials and have received much interest for applications as light-emitting and laser diodes in the blue and green frequency range.

Yoshizawa et al. $[1,2]$ first synthesized GaN nanocolumns on (0001) $\mathrm{Al}_{2} \mathrm{O}_{3}$ by rf-plasma-assisted molecular beam epitaxy (rf-MBE) through a selforganization process. Kikuchi et al. [3] reported a fabrication of GaN-nanocolumn-based light-emitting diodes (LEDs) on n-type Si substrates.

In spite of remarkable progresses in the growth of GaN nanostructures and their application to optoelectric devices, basic physical properties of the nanostructures still remain poorly studied, in particular the electric transport phenomena. It is important to study the electric transport properties of GaN nanocolumns to evaluate them as the fundamental building blocks for not only optoelectronic devices but also electronic ones. Moreover, the nanocolumns are expected to show novel phenomena in the transport mechanism.

We study the electric resistivity in single GaN nanocolumns, and report the impurity-conduction, the nonlinear conduction, and the magnetoresistance effect in this paper.

*This paper was presented at the 6th International Symposium on Surface Science - Towards Nano, Bio and Green Innovation-, Tower Hall Funabori, Tokyo, Japan, December 11-15, 2011.

${ }^{\dagger}$ Corresponding author: t-sekine@sophia.ac.jp

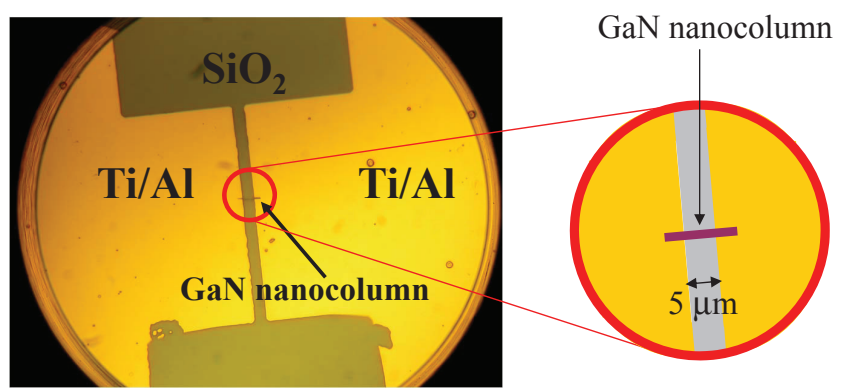

FIG. 1: Photograph (left panel) and the schematic (right panel) of a single GaN nanocolumn with $\mathrm{Ti} / \mathrm{Al}$ contact electrodes. The gap between the $\mathrm{Ti} / \mathrm{Al}$ electrodes is $5 \mu \mathrm{m}$.

\section{EXPERIMENTAL}

The n-type GaN nanocolumns were self-organized with a column diameter of about $600 \mathrm{~nm}$ and a length of about $10 \mu \mathrm{m}$ on n-type (111) Si substrate by rf-MBE method. These are grown along the $c$ crystal axis at high density of about $10^{9}-10^{10}$ columns $/ \mathrm{cm}^{2}$. The carrier densities are less than $10^{17} \mathrm{~cm}^{-3}$ in the undoped samples, which was verified by Raman scattering from a plasmon-LO phonon coupled mode [4].

We attach $\mathrm{Ti} / \mathrm{Al}$ electrodes to a single GaN nanocolumn on the substrate by using photolithography as follows: an assemblage of GaN nanocolumns on the growth substrate is sonicated in dichloroethane solution, and the individual GaN nanocolumns are dispersed onto $\mathrm{SiO}_{2}(\sim 300 \mathrm{~nm}$ thickness $) / \mathrm{Si}$ substrate by using the spin coat. The photoresist film is covered on a single GaN nanocolumn. Moreover, the photoresist on the electrodes is removed by conventional photolithography technique. Then the liftoff is done after $\mathrm{Ti}(\sim 30 \mathrm{~nm})$ and $\mathrm{Al}(\sim 400$ $\mathrm{nm})$ are evaporated as electrodes. Finally, the sample is annealed at $600-750{ }^{\circ} \mathrm{C}$ in a minute in a mixed atmosphere of $\mathrm{H}_{2}(20 \%)$ and $\mathrm{Ar}(80 \%)$ gases, and we succeed in creating the ohmic contacts. The gap of the photomask, i.e., 


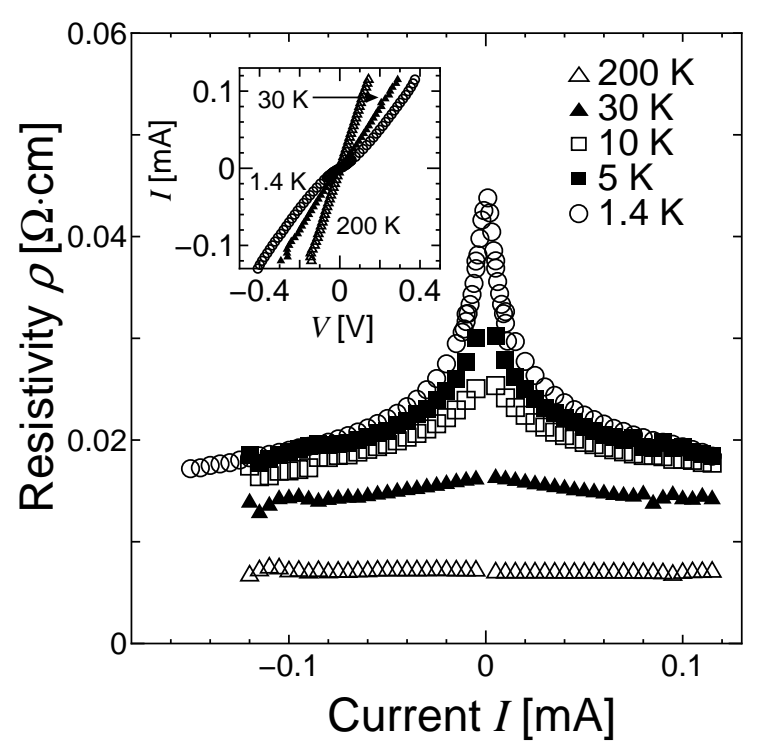

FIG. 2: $\rho-I$ characteristics with DC currents in a single GaN nanocolumn at various temperatures. The inset shows the $I-V$ curves at $1.4,30$, and $200 \mathrm{~K}$.

the length $\ell$ between the electrodes is $5 \mu \mathrm{m}$, as shown in the photograph of Fig. 1.

We also attached $\mathrm{Ti} / \mathrm{Au}$ electrodes to a single GaN nanocolumn, but we could not succeed in creating the ohmic contacts. We measure the electric resistivity along the $c$ axis from room temperature to $1.4 \mathrm{~K}$ in the twoprobe method with constant DC currents and rectangular pulse currents of $10-\mathrm{Hz}$ frequency (Keithley 6221). In the magnetoresistance measurement, we apply magnetic field $B$ up to 12 T normal to the $c$ axis using a superconducting magnet (Oxford Instruments, Teslatron S14/16).

\section{RESULTS AND DISCUSSION}

Figure 2 shows resistivity-current $\rho-I$ characteristics with constant DC currents. We obtained almost the same resistivity as each other with positive and negative currents, indicating that the ohmic contacts are formed. However, we clearly observed the nonlinear conduction at low temperatures. The nonlinearity becomes weaker with increasing temperature $T$. It disappeared at $200 \mathrm{~K}$. The resistivity of the single GaN nanocolumn is $7.2 \times 10^{-3}$ $\Omega \cdot \mathrm{cm}$ at $200 \mathrm{~K}$. Almost the same magnitudes of resistivity are obtained in two other samples. This value is about ten times as small as the resistivity of bulk GaN epitaxial film on sapphire substrate [6].

In order to study the nonlinear conduction we measure the $\rho-I$ characteristics with rectangular pulse currents of which widths are $1.5,3.0$ and $12 \mathrm{~ms}$ with a $10-\mathrm{Hz}$ frequency, as shown in Fig. 3. The nonlinear conduction was observed with rectangular pulse and DC currents at low temperatures and the resistivity is independent of the pulse width, indicating clearly that the nonlinear conduction is not due to the effect of Joule heat but the intrinsic effect of the GaN nanocolumn. Moreover, the wave-form of the rectangular pulse was not transformed, which indicates that the measuring circuit includes no electric capacitance. The nonlinear conduction was observed with

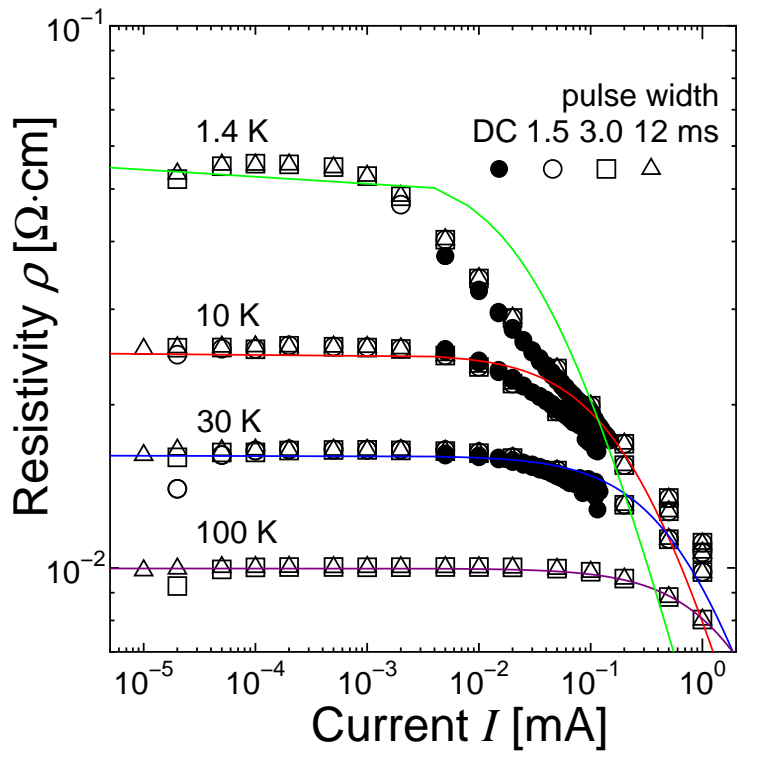

FIG. 3: $\rho-I$ characteristics with pulse currents of 1.5, 3.0 and 12 -ms widths together with those by DC currents in a single GaN nanocolumn at various temperatures. The colored lines denote the fittings of Eq. (2).

large currents, but we found that the ohm's law was kept at small currents. The current where the nonlinear conduction begins to appear increases as the temperature $T$ is increased.

The nonlinear conduction was also observed in GaN nanowires by Kim et al. [7] and in other nanowires [8, 9] and carbon nanotubes [10]. Kim et al. stated that it originated from the formation of the Schottky barriers between the electrodes and sample in the metal-semiconductor junction. In this case, the transport are described by thermionic-emission, diffusion and tunneling processes, and the electric current $I$ as a function of the applied electric voltage $V$ is given as[11]

$$
I=I_{0}\left[\exp \left(\frac{e V}{\eta k_{\mathrm{B}} T}\right)-1\right],
$$

where $e$ is the elementary charge. This equation expresses the thermionic-emission process or the diffusion one when $\eta=1$, and it does the tunneling process when $\eta>1$ and $\eta$ is close to unity. Then, the resistivity $\rho$ against current $I$ is expressed as

$$
\rho=\frac{\eta k_{\mathrm{B}} T S}{e I \ell} \ln \frac{I+I_{0}}{I_{0}}
$$

where $\ell(=5.0 \mu \mathrm{m})$ is the length of the sample and $S$ the area of the cross section. The colored lines in Fig. 3 show Eq. (2) but these do not fit the experimental data in the nonlinear-resistivity region at low temperatures. Moreover, we obtain in the fittings that $\eta=1.7-0.4 \times 10^{3}$ between 1.4 and $100 \mathrm{~K}$, where $\eta$ is much larger than unity. We think that the formation of the Schottky barriers does not explain the nonlinear resistivity.

In general, the applied electric fields become very high in the resistivity measurement of the semiconductor nanostructures, because the distance between their electrodes is very short. Then, the observed nonlinear conduction is probably due to the avalanche breakdown, [11, 


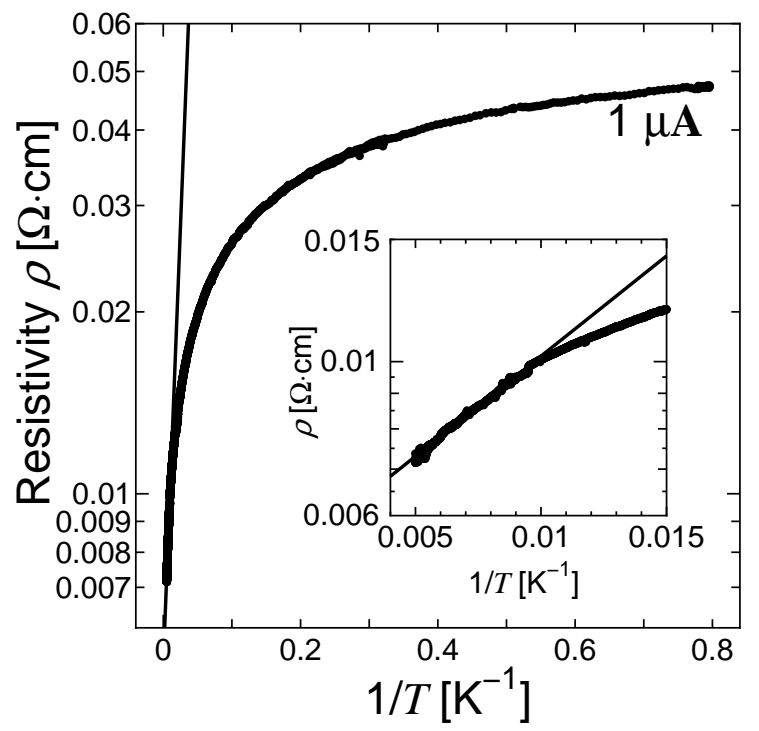

FIG. 4: Arrhenius plot of resistivity $\rho$ in a single GaN nanocolumn when $I=1 \mu \mathrm{A}$. In the inset, it is expanded in the hightemperature region. The solid lines denote a fitting function of $\exp \left(\varepsilon_{d} / 2 k_{\mathrm{B}} T\right)$.

$12]$ i.e., the multiplication of carriers by ionization of impurity levels in a current of energetic electrons, because the threshold of breakdown field increases with rising temperature $[11,13]$.

We studied the temperature dependence of resistivity $\rho$ in a single GaN nanocolumn between 1.4 and $200 \mathrm{~K}$ with a constant current of $1 \mu \mathrm{A}$. Figure 4 shows the Arrhenius plot of resistivity. Figure 3 shows that the ohm's law is kept down to $1.4 \mathrm{~K}$ below $1 \mu \mathrm{A}$. The temperature dependence of resistivity represents an activation-type one of $\exp \left(\varepsilon_{d} / 2 k_{\mathrm{B}} T\right)$ with an activation energy $\varepsilon_{d}=11 \mathrm{meV}$, which is due to donor levels, probably originating from silicon impurities with $\varepsilon_{d}=12-17 \mathrm{meV}$ [5].

At low temperatures, it deviates from the Arrhenius behavior. When many electrons are localized at impurities at low temperatures, the electric transport would be governed by phonon-assist hopping conduction, e.g., by conduction in an impurity band of localized states with random spatial and energy distributions.

The variable-range hopping conduction is given by [14$16]$

$$
\rho=\rho_{0} \exp \left[\left(\frac{T_{0}}{T}\right)^{\beta}\right],
$$

where $T_{0}$ is the characteristic temperature. The exponent $\beta$ depends on different hopping conductivity mechanism: $\beta=1 /(1+d)$ at relatively higher temperatures, known as Mott variable-range hopping, where $d$ is the dimensionality, and $\beta=1 / 2$ at very low temperatures, known as Efros-Shklovskii variable-range hopping. The $T_{0}$ is given as

$$
k_{\mathrm{B}} T_{0}=\frac{2^{9}}{9 \pi} \frac{1}{N\left(E_{\mathrm{F}}\right) \xi^{3}},
$$

in the Mott variable-range hopping, where $\xi$ is the localization length and $N\left(E_{\mathrm{F}}\right)$ the density of states at the

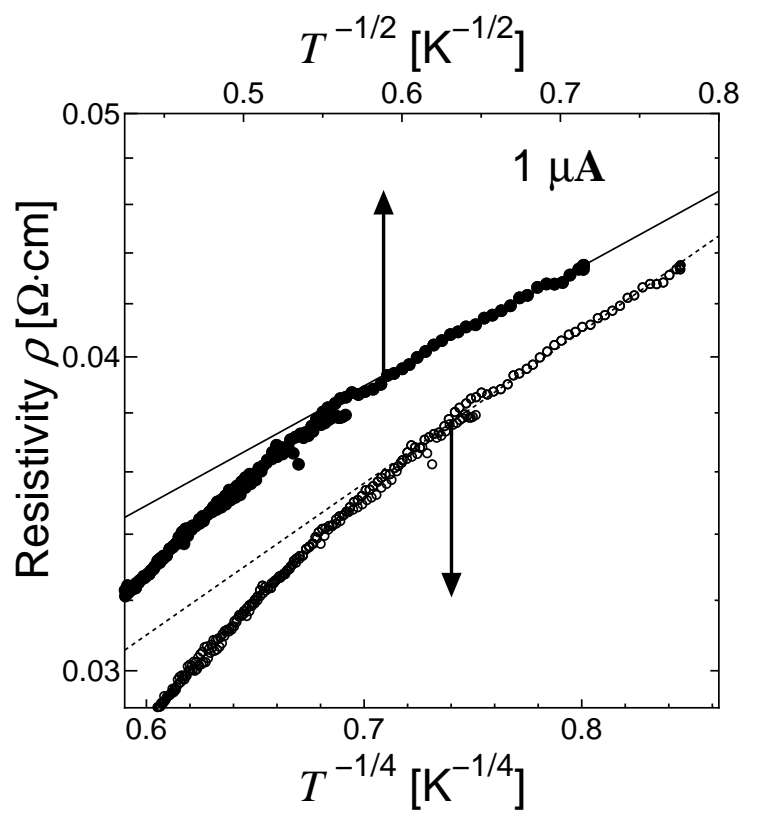

FIG. 5: Temperature dependence of $\log \rho$ as functions of $T^{-1 / 4}$ and of $T^{-1 / 2}$ in a single GaN nanocolumn. The dotted and solid lines denote Eq. (3) when $\beta=1 / 4$ and $1 / 2$, respectively.

Fermi level, and

$$
k_{\mathrm{B}} T_{0}=\frac{8 e^{2}}{\kappa \xi},
$$

in the Efros-Shklovskii variable-range hopping, where $\kappa$ is the static dielectric constant.

It holds that $d=3$ in the single GaN nanocolumn, because the diameter of about $600 \mathrm{~nm}$ is much larger than the Bohr radius of donor level. Figure 5 shows $\log \rho$ as functions of $T^{-1 / 4}$ and of $T^{-1 / 2}$ at very low temperatures. It is seen that the Mott variable-range hopping is favored in a wider temperature range, which indicates that the resistivity $\rho$ below about $5 \mathrm{~K}$ is governed by it. We obtain $T_{0}=4.3 \mathrm{~K}$ in the fitting and consequently estimate $\xi \sim$ $130 \mathrm{~nm}$ from Eq. (4). Here, we assume that the donor density $N$ is approximately $10^{17} \mathrm{~cm}^{-3}$ and the width of the impurity band due to donor levels is approximately $5 \mathrm{meV}$, resulting in $N\left(E_{\mathrm{F}}\right) \sim 2 \times 10^{16} /\left(\mathrm{cm}^{3} \cdot \mathrm{meV}\right)$. The obtained $\xi$ is close to the average distance between donor impurities, $N^{-1 / 3} \sim 22 \mathrm{~nm}$. The Mott variable-range hopping was also observed in Cr-doped GaN films [17] and InN nanowires [18].

On the other hand, the Efros-Shklovskii variable-range hopping would fit with the experiment below $3.4 \mathrm{~K}$, assuming $T_{0}=0.63 \mathrm{~K}$. We estimate that $\xi \sim 24 \mu \mathrm{m}$ by using $\kappa=9$ [19], which is much larger than the diameter of sample.

In the intermediate temperature region above about 10 $\mathrm{K}$, we think that the nearest-neighbor hopping conduction, i.e., the Miller-Abrahams type one mainly occurs. It is written as $\rho \propto \exp \left(\varepsilon_{3} / k_{\mathrm{B}} T\right)$, where $\varepsilon_{3}$ is the hopping energy [14-16, 20].

Figure 6 shows the magnetoresistivity ratio $\{\rho(B)-$ $\rho(0)\} / \rho(0)$ below $12 \mathrm{~T}$. We found a strong positive magnetoresistance at low temperatures, namely, an increase of resistivity by a factor of 1.3 was observed at $12 \mathrm{~T}$ when 


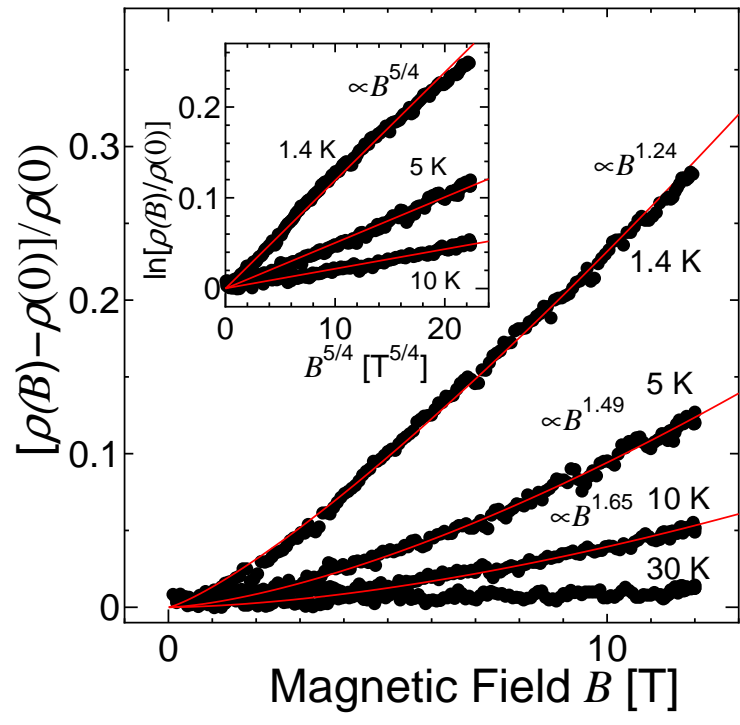

FIG. 6: Magnetoresistivity ratio of a single GaN nanocolumn at various temperatures when $I=1 \mu \mathrm{A}$. The inset shows $\ln \{\rho(B) / \rho(0)\}$ as a function of $B^{5 / 4}$. The red lines denote the fittings.

$T=1.4 \mathrm{~K}$. However, it gradually weakened with increasing temperature, and disappeared above $30 \mathrm{~K}$.

In the bulk GaN films, the temperature dependences of Hall effect and resistivity were explained by two-band model, i.e., two kinds of electrons in the conduction band and the impurity band [21-23]. Similar analysis was done in p-type GaN [24]. In the two-carrier model the magnetoresistivity ratio is expressed as [12]

$$
\frac{\rho(B)-\rho(0)}{\rho(0)}=\frac{C_{3} B^{2}}{C_{1}+C_{2} B^{2}},
$$

where $C_{1}, C_{2}$, and $C_{3}$ are constant. When the magnetic field $B$ is weak, the magnetoresistivity ratio is proportional to $B^{2}$. However, the present result indicates that it is proportional to $B^{1.24}$ at $1.4 \mathrm{~K}$ and the exponent increases with increasing temperature, which is not understood by the simple two-carrier model.

In the variable-range hopping conduction regime, two kinds of mechanism are considered for magnetoresistance: one is the positive magnetoresistance and the other is the negative magnetoresistance due to reduction of the Anderson localization. In a magnetic field the wave functions of impurity electrons are squeezed in the transverse direction, resulting in reduction of the hopping between impurity levels, i.e., positive magnetoresistance. The pos- itive magnetoresistance was observed in temperature region of the variable-range hopping in doped semiconductors, which exponentially increases as a function of magnetic field [16]:

$$
\rho(B)=\rho(0) \exp \left(C_{0} B^{\gamma}\right),
$$

where $C_{0}$ depends on $T$ but does not on $B$. It holds that $\gamma=2$ in the weak-field limit of $B \ll B_{c}$, while $\gamma=1 / 3$ in the strong-field limit of $B \gg B_{c}$, the characteristic field $B_{c}$ being given as

$$
B_{c}=\frac{N^{1 / 3} c \hbar}{a e}
$$

where $c$ is the light velocity and $a$ is the state radius in the absent of a magnetic field and given as $\hbar / \sqrt{2 m^{*} \varepsilon_{d}}$. The effective mass of electron $m^{*}$ is reported to be $0.2 \times m_{0}, m_{0}$ being the electron mass [19]. This equation corresponds to

$$
\frac{\lambda^{2}}{a}=N^{-1 / 3}
$$

where the magnetic length is given as $\lambda=\sqrt{c \hbar / e B}$. The inset of Fig. 6 shows $\ln \{\rho(B) / \rho(0)\}$ as a function of $B^{5 / 4}$. The magnetoresistance is well expressed by Eq. (7) when $\gamma=5 / 4$. We think that this value of $\gamma$ is plausible in the present case of $B_{c} \sim 7.4 \mathrm{~T}$.

\section{CONCLUSION}

In conclusion, we succeeded in creating the ohmic contacts to a single GaN nanocolumn on $\mathrm{SiO}_{2} / \mathrm{Si}$ substrate by using photolithography. We observed the nonlinear conduction, which probably originates from the avalanche breakdown in the nanostructures. The temperature dependence of resistivity represented the activation-type one with an activation energy of $11 \mathrm{meV}$ due to donor levels at high temperatures. At low temperatures, it showed the Mott variable-range hopping conduction. It was found that this conduction represented strong positive magnetoresistance of $\ln \{\rho(B) / \rho(0)\} \propto B^{5 / 4}$.

\section{Acknowledgments}

We are indebted to H. Atari and Y. Hayashi for their technical assistance.
[1] M. Yoshizawa, A. Kikuchi, M. Mori, N. Fujita, and K. Kishino, Inst. Phys. Conf. Ser. 155, 187 (1997).

[2] M. Yoshizawa, A. Kikuchi, M. Mori, N. Fujita, and K. Kishino, Jpn. J. Appl. Phys. 36, L459 (1997).

[3] A. Kikuchi, M. Kawai, M. Tada, and K. Kishino, Jpn. J. Appl. Phys. 43, L1524 (2004).

[4] T. Sekine, K. Muramoto, S. Suzuki, H. Kuroe, H. Sekiguchi. A. Kikuchi, and K. Kishino, Proc. the XXIst Int. Conf. on Raman Spectroscopy ed. by R. Withnall and B. Z. Chowdhry (IM Publications, West Sussex, 2008) p.
603.

[5] W. Götz, N. M. Johnson, C. Chen, H Liu, C. Kuo, and W. Imler, Appl. Phys. Lett. 68, 3144 (1996).

[6] M. Ilegems and H. C. Montgomery, J. Phys. Chem. Solids. 34, 885 (1973).

[7] J.-R Kim, H. M. So, J. W. Park, J.-J. Kim, J. Kim, C. J. Lee, and S. C. Lyu, Appl. Phys. Lett. 80, 3548 (2002).

[8] J.-Y Yu, S.-W. Chung, and J. R. Heath, J. Phys. Chem B 104, 11864 (2000).

[9] Y.-F. Lin, W.-B Jian, C. P. Wang, Y.-W. Suen, Z.-Y. 
Wu, F.-R. Chen, J.-J. Kai, J.-J. Lin, and J.-J Lin, Appl. Phys. Lett. 90, 223117 (2007).

[10] C. Zhou, J. Kong, and H. Dai, Appl. Phys. Lett. 76, 1597 (2000).

[11] S. M. Sze and K. K. Ng, Physics of Semiconductor Devices (Jhon Wiley \& Sons, Hoboken, 2007).

[12] J. M. Ziman, Principles of the Theory of Solids (Cambridge University, Cambridge, 1972).

[13] C. Y. Chang, S. S. Chiu, and L. P. Hsu, IEEE Trans. Electron Devices 18, 391 (1971).

[14] N. F. Mott and E. A. Davis, Electronic Processes in NonCrystalline Materials, (Clarendon, Oxford, 1979) p. 50.

[15] N. F. Mott, Metal-Insulator Transitions (Taylor \& Fancis, London, 1990) p.50.

[16] B. I. Shkolovskii and A. L. Efros, Elelctronic Properties of Doped Semiconductors in Springer Series in Solid-State
Sciences Vol. 45 (Springer-Verlag, Berlin, 1984) p. 202.

[17] S. Y. Wu and N. Newman, Appl. Phys. Lett. 89, 142105 (2006).

[18] L. H. Haung, D. Li, P. Chang, S. Chu, H. Bozler, I. S. Beloborodov, and J. G. Lu, Phys. Rev. B 83, 245310 (2011).

[19] H. Harima, J. Phys.: Condens. Matter 14, R967 (2002).

[20] A. Miller and E. Abraham, Phys. Rev. 120, 745 (1960).

[21] D. C. Look and M. J. Molnar, Appl. Phys. Lett. 70, 3377 (1997).

[22] D. C. Look and J. R. Sizelove, Phys. Rev. Lett. 82, 1237 (1999).

[23] H. Eshghi, D. Lancefield, B. Beaumont, and P. Gibart, Phys. Stat. Sol. (b) 216, 733 (1999).

[24] D. Lancefield and H. Eshghi, J. Phys.: Condens. Matter 13, 8939 (2001). 